\title{
Intergenomic interactions affect female reproduction: evidence from introgression and inbreeding depression in a haplodiploid mite
}

\author{
M-J Perrot-Minnot ${ }^{1}$, A Migeon ${ }^{2}$ and M Navajas ${ }^{2}$ \\ ${ }^{1}$ Equipe Ecologie Evolutive, UMR CNRS 5561 Biogéosciences, Université de Bourgogne, 6 blvd Gabriel, 21000 Dijon, France; ${ }^{2}$ Institut \\ National de la Recherche Agronomique, CBGP, Campus international de Baillarguet, CS 30016, 34988 Montferrier sur Lez, France
}

\begin{abstract}
Nuclear and cytoplasmic genomes can coevolve antagonistically or harmoniously to affect fitness. One commonly used test for nuclear-cytoplasmic coadaptation relies on the breakup of coadapted gene complexes by introgression, potentially resulting in an increased frequency of nuclear alleles in deleterious interaction with an alien cytoplasm. We investigated the phenotypic effect of such genes on female reproduction in outbred and inbred introgressed lines of the haplodiploid mite Tetranychus urticae. Introgression changed female lifetime fecundity and increased male production, in ways suggesting a control of fecundity by nuclear genes. Conversely introgression reduced the fertilization rate,
\end{abstract}

possibly due to sperm-egg incompatibility or maternal effects. The intensity of inbreeding depression expressed as a reduction in fecundity was more severe in introgressed females than in nonintrogressed ones, giving evidence for recessive interacting alleles contributing to residual nucleocytoplasmic incompatibility. Overall, our data suggest recessive negative interactions between nuclear and cytoplasmic genes. This study is the first report of a contribution of nuclear polymorphism within a population to deleterious interactions with an alien cytoplasmic genome.

Heredity (2004) 93, 551-558. doi:10.1038/sj.hdy.6800552 Published online 25 August 2004

Keywords: nuclear-cytoplasmic interaction; hybrid breakdown; inbreeding depression; haplodiploidy; mite; tetranychidae

\section{Introduction}

Nuclear and cytoplasmic genomes have functional interactions through the peptides they encode, contributing to a common physiological pathway (for a review, see Blier et al, 2001; Sackton et al, 2003). Coevolution between nuclear and cytoplasmic genes is thus expected to occur and select for coadapted gene complexes. Plant cytonuclear interactions have been the most thoroughly described over the last few decades, especially cytoplasmic male sterility. By contrast, only few studies have reported such nuclear-cytoplasmic interactions in animals. Evidence for intergenomic coadaptation comes from molecular data showing non-neutral evolution of mtDNA with respect to its nuclear background (for a review, see Blier et al, 2001). Recently, evidence of interaction between mitochondrial and nuclear genes in cognitive functioning was demonstrated experimentally in mice (Roubertoux et al, 2003). Coadaptation between nuclear and cytoplasmic genomes has also been reported in crosses that display hybrid breakdown in invertebrates, between genetically divergent populations or at the interspecific level (Breeuwer and Werren, 1995; Edmands and Burton, 1999; Willett and Burton, 2001; Sackton et al, 2003). Cytonuclear coadaptation was evidenced in the disruption of cytochrome $c$ oxidase

Correspondence: M-J Perrot-Minnot, Equipe Ecologie-Evolution (UMR CNRS 5561 Biogéosciences), Université de bourgogne, 6 Blvd Gabriel, 21000 Dijon, France. E-mail: mjperrot@u-bourgogne.fr

Received 27 November 2003; accepted 11 June 2004; published online 25 August 2004
(COX) activity following backcrosses of divergent intraspecific lines in the copepod Tigriopus californicus (Edmands and Burton, 1999; Willett and Burton, 2001) and in Drosophila simulans (Sackton et al, 2003). At the interspecific level, the role of cytonuclear interactions in the evolution of hybrid incompatibility has been revealed in the contribution of disrupted interactions between nuclear and cytoplasmic genes to hybrid breakdown (Breeuwer and Werren, 1995). Progressive heterospecific introgression of a nuclear genome into a cytoplasmic background it has not coevolved with did not reduce hybrid breakdown (Breeuwer and Werren, 1995) (Table 1, (1)). However, if deleterious nuclear-cytoplasmic interactions involve recessive nuclear alleles, progressive introgression could rescue hybrids and increase fitness (Table 1, (3)). This type of nuclear-cytoplasmic incompatibility caused by negative interactions between recessive nuclear alleles and cytoplasmic genes may be overlooked in hybridization and introgression experiments, since they can only be detected in haploid, hemizygous or homozygous (inbred) individuals in introgressed lines. We have developed the latter approach to test for intergenomic coadaptation in a haplodiploid mite species. Haplodiploidy is of great interest in such studies because chronic inbreeding occurs in many haplodiploid species (Werren, 1993), including phytophagous mites (Helle and Overmeer, 1973). In these species, inbreeding depression is expected to be low in the original cytoplasmic background, because recessive nuclear genes are continuously purged, including those potentially interacting with cytoplasmic genes. However, such deleterious recessive alleles may be more frequent in a 
Table 1 Fitness values of genotypes interacting with an alien cytoplasmic genome relative to fitness values in the original cytoplasm, depending on the type of interaction between nuclear genotype and an alien cytoplasm, with allele A completely dominant over B

\begin{tabular}{lccclll}
\hline Cytoplasm & \multicolumn{3}{c}{ Nuclear genotypes } & & \multicolumn{2}{c}{ Mean fitness of } \\
\cline { 2 - 3 } & $A A$ & $A B$ & $B B$ & & Introgressed lines compared to parental lines & Inbred introgressed lines vs outbred introgressed lines \\
\hline Original & 1 & 1 & 1 & & - & Not significantly different \\
Alien (1) & $1-s$ & $1-s$ & $1-s^{\prime}$ & & Strongly decreased & - \\
Alien (2) & $1-s$ & $1-s$ & 1 & & Not significantly different & Not significantly different \\
Alien (3) & 1 & 1 & $1-s^{\prime \prime}$ & & Not significantly different & Decreased \\
\hline
\end{tabular}

(1) Negative interactions between all alleles and cytoplasmic genes: introgression fails to rescue hybrids, (2) negative interactions between allele A and cytoplasmic genes: introgression rescues recessive homozygotes only and eliminates the deleterious interacting allele A, (3) negative interactions between allele B and cytoplasmic genes: introgression will not eliminate the deleterious interacting allele B.

In (2) and (3), introgression has increased mean fitness of hybrids up to the fitness of parental strains.

an an inbred population at equilibrium, we expect the frequency of deleterious alleles to be very low. Thus, most genotypes adapted to the original cytoplasmic genome are expected to have equal fitness.

nuclear genome that has not coevolved with the cytoplasmic background. In addition, inbreeding depression in haplodiploids is expected to affect mainly 'female-limited' traits which are not exposed to selection in hemizygous males (Crozier, 1985; Werren, 1993; Saito et al, 2000). This can limit the practical difficulties raised from loss of lines and incidental selection on viability as inbreeding proceeds.

We tested for cytonuclear interactions by hybridization and introgression between two strains of the spider mite Tetranychus urticae from geographically distant areas (the Netherlands and Greece). Inbreeding was performed in introgressed lines and control strains to test whether negative nuclear-cytoplasmic interactions could involve recessive nuclear alleles (Table 1, (3)). No genetic polymorphism was found between the two strains studied, when comparing sequences of the nuclear ITS gene (Navajas et al, 2000). However, it is probable that their mtDNA exhibits some polymorphism, based on the COI gene sequence of another Greek strain showing $4.5 \%$ divergence from the Dutch strain used in this study (Navajas et al, 1998). The two strains also harbored Wolbachia endosymbionts, but infection had no effect on reproductive parameters and was not inducing cytoplasmic incompatibility (Perrot-Minnot et al, 2002). Several components of female fitness were measured following introgression and inbreeding: lifetime fecundity, oviposition rate, embryonic mortality, and sex ratio. Our data are discussed in the light of predicted changes in female relative fitness following introgression and inbreeding, under the hypothesis of a breakup of co-adapted nucleocytoplasmic genomes.

\section{Material and methods}

\section{Strains and culture}

Two strains of T. urticae were used: mites collected from Rose bay (Nerium oleander) in Athens, Greece (further labelled G), and mites collected from Sambucus sp in the Netherlands (further labelled U). At the time of the experiments, the former had been reared for 2 years on detached leaves of kidney bean Phaseolus vulgaris (var. Contender) in closed and aerated containers in a climate room at $21^{\circ} \mathrm{C}, 16: 8 \mathrm{~L}: \mathrm{D}$, and $60-70 \%$ relative humidity. Population size was maintained at approximately 50 to $>500$ adults. The latter strain had been mass-reared on kidney bean in a greenhouse for more than 10 years in Montpellier, at a population size of a hundred to several thousands. Both strains were partially reproductively isolated, with hybrid dysgenesis resulting in approx. $80 \%$ decrease in fecundity and more than $90 \%$ offspring mortality in F1 females (Perrot-Minnot and Migeon, unpublished data). Hybrids' viability and fecundity were increased within four generations of backcrosses up to levels comparable to the respective paternal strain, excepting a lower proportion of females in progeny from $\mathrm{G}$ females introgressed with $\mathrm{U}$ nuclear genome. Thus, hybrid breakdown was mainly caused by negative nuclear-nuclear interactions found in both reciprocal hybrid crosses (Perrot-Minnot and Migeon, unpublished). Dominant alleles negatively interacting with cytoplasmic genes (if any) would have been purged during the process of introgression (Table $1,(1)$ ). The possibility that negative recessive nuclear-cytoplasmic interactions contribute to hybrid breakdown (Table 1, (3)) was investigated in the present study by performing inbreeding in introgressed lines. The terminology used for introgressed lines indicates the cytotype in brackets: lines with $U$ nuclear genome and G cytotype are designated as U[G], with $U$ and $G$ being the two parental lab strains.

\section{Introgression lines}

Viable and fertile F2 females produced by G grandmothers were used to initiate isofemale lines backcrossed to males from the paternal strain U. At each generation, two to 10 virgin females per family were mated to virgin $\mathrm{U}$ males (from stock culture) and then isolated. From the sixth to the 10th generation, one female was kept per line and approximately 70 lines were maintained, stemming from five original $\mathrm{G}$ females mated to $\mathrm{U}$ males. In theory, each backcross generation has replaced half of the residual maternal genome $G$ with the paternal genome $\mathrm{U}$ in the female progeny, whereas the maternally inherited cytoplasmic genome has not mixed. Thus, $97 \%$ of the original maternal genome $\mathrm{G}$ would have been replaced by the paternal genome after five introgression generations and $99.9 \%$ after 10 generations, assuming Mendelian segregation and inheritance. Female reproduction on the first week of oviposition was monitored every generation from the sixth to the 15th introgression generation, and every five generations thereafter. A total of 45 isofemale lines were taken at random at the 15th 
generation and maintained thereafter, among which 33 lines reached the 34 th generation. Female reproductive parameters were very stable from the 10th introgression generation (data not shown). This suggests that selection on viability and fertility incidentally occurring from line maintenance could be neglected, once nuclear-nuclear incompatibilities involved in hybrid breakdown were removed by introgression.

\section{Crossing procedures}

For experimental crosses and isofemale line maintenance, males and females were virgin upon mating. Female teliochrysalids, the last developmental stage before adult emergence, were individually isolated from the rearing arena of the previous generation on a $2-\mathrm{cm}-$ diameter disk of fresh bean leaf placed on a water-soaked cotton layer. Upon emergence, each female was presented with two virgin males produced in mass by virgin females isolated as teliochrysalids from $U$ stock culture. Males were discarded 2 days later, and mated females were allowed to oviposit for 6 days. To measure the female lifetime offspring production, females were transferred to a fresh rearing arena every week. Eggs were scored and adult emergence was checked regularly. Offspring were sexed upon sexual differentiation (deutonymphal stage) and removed to minimize overexploitation of the leaf disk. We estimated mortality rate as the difference between the number of eggs recorded and the number of adults emerged, weighted by the number of eggs recorded. Preliminary observations have shown that mortality occurred mostly at the embryonic stage, but shrivelling of aborted eggs made them difficult to score. Since our rearing conditions prevented escapes from larva eclosion to adult emergence, recording eggs and adults afforded a reliable estimate of mortality. Sex ratio was calculated as the proportion of females among adult offspring, that is, the secondary sex ratio.

\section{Effect of introgression on female reproduction}

Since introgressed isofemale lines were derived from two parental strains with contrasting reproductive patterns, we first document the effect of introgression of $U$ genome into $\mathrm{G}$ cytoplasm on female lifetime reproduction. This effect was assessed after 34 generations of repeated backcross in isofemale U[G] lines. As sex allocation in haplodiploid species can be affected by sperm availability, since unfertilized eggs develop into adult males, sperm depletion can potentially be a confounding factor in the analysis of lifetime female reproduction. Thus, we performed two types of crosses: both U[G] females and control $U$ females from stock culture were either mated once, or were given access to multiple mating by replacing their mates with two virgin males every week. These mating treatments will be referred as monogamous and polyandrous females, respectively. To avoid pseudoreplication, only one female per introgression line was used in each mating treatment.

\section{Response of introgressed lines to inbreeding}

Brother-sister mating was started in 30 U[G] lines after 27 introgression generations, to assess the effect of inbreeding on female reproduction. From the first to the 10th generation of full-sib mating, offspring production over the first six oviposition days and egg viability were measured, to monitor the change in female reproduction with increasing inbreeding level. In parallel, 20 inbred isofemale lines were established from $U$ females from the stock culture to set up a control for inbreeding depression in the original cytoplasmic background. After six and five inbreeding generations in $\mathrm{U}[\mathrm{G}]$ and $U$ lines, respectively, we measured the intensity of inbreeding depression on lifetime female reproduction. To avoid pseudoreplication, only one female per inbred line was used.

\section{Statistical analysis}

Offspring production (total number of eggs, total number of adult males, and females produced) and length of oviposition period were analyzed using parametric statistics. Two-factor ANOVAs were performed to test the effects of female type, mating treatment (monogamous vs polyandrous), and interaction of both (introgression experiment), and the effects of female type, reproductive status (inbred vs outbred), and interaction of both (inbreeding experiment). The TukeyKramer test for a posteriori multiple pairwise comparisons and the Student's t-test were used for mean comparisons (Jump 3.2.2. software, SAS Institute Inc.). Sex ratio and mortality rate were analyzed using nonparametric tests (Kruskall-Wallis analysis and $a$ posteriori multiple comparison test; Siegel and Castellan, 1988). A Bonferroni correction for multiple comparisons was used to account for the nonindependence of the tests performed for each reproductive parameter, by using a significance level of $\alpha^{\prime}=\alpha / k$ for $k$ comparisons (Sokal and Rolf, 1995).

\section{Results}

\section{Effect of introgression on female reproduction}

The effect of introgression of $U$ genome into $G$ cytoplasm on lifetime female reproduction was measured after 34 generations of repeated backcrosses in isofemale lines and compared to the original $U$ and $G$ parental strains. The degree of polyandry did not affect the reproductive parameters measured in U[G], U, and G females (Table 2). Since no effect of mating treatment and interaction (type of female $\times$ mating treatment) was significant in twofactor ANOVAs, data from monogamous and polyandrous females were pooled within each type of cross, to perform univariate ANOVAs and post hoc pairwise comparisons. Introgression increased the mean lifetime offspring production of U[G] females by approximately $90 \%$ compared to the maternal strain G. Egg production in U[G] females and U females was similar, as well as estimated embryonic or larval mortality (Table 2). The higher fecundity of introgressed females and $U$ females compared to $\mathrm{G}$ females was only partially explained by a significantly longer oviposition period (Table 2). Daily oviposition rate (estimated as the ratio of lifetime fecundity to the length of oviposition period in days) was significantly higher in U[G] and $U$ females compared to $G$ females $\left(F_{2,160}=27.5, P<0.001\right)$, with a mean \pm SD number of eggs laid per day of $8.9 \pm 2.5$, $8.1 \pm 2$ and $6.1 \pm 1.5$ in $U[G], U$ and $G$ females, respectively. In addition, increased lifetime fecundity in U[G] females translated exclusively into the production of more males, with $90 \%$ of the increase in offspring 
production being of the male sex, the remaining $10 \%$ may correspond to residual embryonic mortality. Consequently, sex ratio in U[G] offspring was male biased (median (quartiles) of female proportion: 0.39 (0.26-0.52), $n=51$ ), whereas both parental strains exhibited a femalebiased sex ratio ( $\mathrm{U}$ females, $0.58(0.50-0.72), n=55 ; \mathrm{G}$ females, $0.74(0.67-0.80), n=57)$. Interestingly, the introgressed U[G] females produced the same number of female offspring as females from their maternal $G$ strain (Table 2). This pattern of increased lifetime offspring production and decreased sex ratio in introgressed lines evidenced at the 34th generation was already significant for the first 6 days of oviposition after five generations of repeated backcrosses, and remained stable thereafter. Offspring production $(O$, mean $\pm S D)$ and sex ratio $(S$, median (quartiles)) during the first 6 days of oviposition after 5, 10, 15, 20, and 30 generations were in fact comparable to the ones observed in the experimental females at the 34th generation $\left(O_{5}=91.8 \pm 24.5, S_{5}=0.47\right.$ $(0.35-0.58), n=40 ; O_{10}=73.9 \pm 22.9, S_{10}=0.60(0.46-0.73)$, $n=72 ; \quad O_{15}=92.4 \pm 19.7, \quad S_{15}=0.54 \quad(0.44-0.63), \quad n=48$; $\mathrm{O}_{20}=99.9 \pm 18.1, S_{20}=0.53(0.48-0.65), n=44 ; O_{30}=87.6$ $\pm 28.5, \quad S_{30}=0.51 \quad(0.44-0.61), \quad n=42 ; \quad O_{34}=98.3 \pm 8.3$, $\left.S_{34}=0.49(0.39-0.61), n=24\right)$.

\section{Response of introgressed lines to inbreeding}

We analyzed the change in female reproduction under inbreeding after five (U) or six (U[G]) generations of brother $\times$ sister mating. Reproductive system (inbred $v s$ outbred) and type of female (introgressed U[G] vs control $\mathrm{U})$ were included in two-factor ANOVAs performed on offspring production and length of oviposition period (Table 3). There was a significant effect of inbreeding on lifetime fecundity, and its intensity was different between introgressed and control females, as shown by a significant female $\times$ reproductive system interaction (Table 3). Lifetime offspring production of inbred females was decreased by $40 \%$ in introgressed U[G] females and by $16 \%$ in control $U$ females compared to outbred U[G] and $U$ females, respectively (Table 3 ). Thus, inbreeding depression on fecundity was more severe in introgressed $U[G]$ females than in control $U$ females. This reduction in fecundity was not due to a shorter length of oviposition period (Table 3), suggesting that oviposition rate was reduced. In fact, the estimated daily oviposition rate of inbred females compared to outbred females decreased from $8.4 \pm 2$ to $5.8 \pm 3$ in U[G] and from $8.2 \pm 1.6$ to $7.9 \pm 3.7$ in $U$ females, respectively. In addition, estimated offspring mortality was significantly increased in the progeny of inbred U[G] females, but not of inbred U females, compared to their respective outbred controls (Table 3). No difference in the proportion of female offspring produced was observed between inbred females and outbred females either in U[G] lines or in $U$ ones (Mann-Whitney tests, $U_{24,20}=237$ and $U_{28,16}=179$, respectively): inbred introgressed females produced a sex ratio as male-biased as outbred introgressed females, in contrast with the female-biased sex ratio produced by inbred and outbred $U$ females (Table 3). Thus, reduction of fecundity and egg viability as a consequence of inbreeding in U[G] females affected both sexes similarly.

We assessed whether the intensity of inbreeding depression changed with female age by monitoring 
Table 3 Effect of inbreeding on introgressed U[G] lines and control U strain

\begin{tabular}{|c|c|c|c|c|c|c|c|}
\hline \multirow[t]{2}{*}{ Females $\times U$ male } & \multicolumn{2}{|c|}{$\begin{array}{l}\text { Oviposition period } \\
\text { (length in days) }\end{array}$} & \multirow{2}{*}{$\begin{array}{c}\text { Lifetime fecundity } \\
\text { (number of eggs) } \\
\text { Mean } \pm S D\end{array}$} & \multicolumn{2}{|c|}{$\begin{array}{c}\text { Offspring mortality rate } \\
\text { (proportion) }\end{array}$} & \multicolumn{2}{|c|}{$\begin{array}{c}\text { Sex ratio } \\
\text { (proportion of females) }\end{array}$} \\
\hline & $\mathrm{N}$ & $M e a n \pm S D$ & & $\mathrm{~N}$ & Median (first quart.) & $\mathrm{N}$ & Median (first quart.) \\
\hline $\mathrm{U}[\mathrm{U}]$ outbred $^{\mathrm{a}}$ & 28 & $27.40 \pm 7.74$ & $217.25 \pm 48.18^{*}$ & 28 & $0.09(0.07-0.12)$ & 28 & $0.64(0.54-0.72)$ \\
\hline $\mathrm{U}[\mathrm{U}]$ inbred (F5) & 16 & $26.69 \pm 11.16$ & $182.06 \pm 62.79^{*}$ & 16 & $0.10(0.06-0.21)$ & 16 & $0.55(0.40-0.73)$ \\
\hline U[G] outbred ${ }^{\mathrm{a}}$ & 24 & $30.08 \pm 10.52$ & $237.25 \pm 56.29^{* * *}$ & 24 & $0.10(0.08-0.12)^{* * *}$ & 24 & $0.39(0.23-0.55)$ \\
\hline U[G] inbred (F6) & 23 & $27.13 \pm 11.20$ & $142.30 \pm 71.90^{* * *}$ & 21 & $0.18(0.13-0.29)^{* * *}$ & 20 & $0.33(0.23-0.54)$ \\
\hline \multirow[t]{2}{*}{$\mathrm{U}[\mathrm{G}]$ inbred (F9) } & 19 & $26.10 \pm 11.05$ & $140.78 \pm 61.38$ & 18 & $0.16(0.11-0.32)$ & 18 & $0.31(0.12-0.46)$ \\
\hline & \multicolumn{3}{|c|}{ Two-factors ANOVAs (F ratios) } & \multicolumn{4}{|c|}{ Kruskall-Wallis } \\
\hline Whole model & & $\mathrm{F}_{3,87}=0.5, P=0.67$ & $F_{3,87}=11.5, P<0.0001$ & \multirow{4}{*}{\multicolumn{2}{|c|}{$H=14.2$, d.f. $=3, P<0.003$}} & $H=$ & 2 , d.f. $=3, P<0.001$ \\
\hline Strain effect & & - & $\mathrm{F}_{1,87}=0.4, P=0.44$ & & & & \\
\hline Inbreeding effect & & - & $\mathrm{F}_{1,87}=26.1, P<0.0001$ & & & & \\
\hline Interaction & & - & $\mathrm{F}_{1,87}=5.5, P=0.02$ & & & & \\
\hline
\end{tabular}

Mean \pm SD lifetime oviposition length and offspring production, and median (first quartiles) sex ratio, and estimated mortality rate are given. $N$, number of individual females (one per line). Fx refers to the number of generations of inbreeding (full-sib matings); F9 was not included in the statistical analysis. Student's $t$-test (oviposition period and lifetime fecundity) and Mann-Whitney U-test (ratios) were used to compare outbred and inbred crosses within each type of line $\left({ }^{*} 0.01<P<0.05 ;{ }^{* * *} P<0.001\right)$

aSame crosses as in Table 2, performed simultaneously.
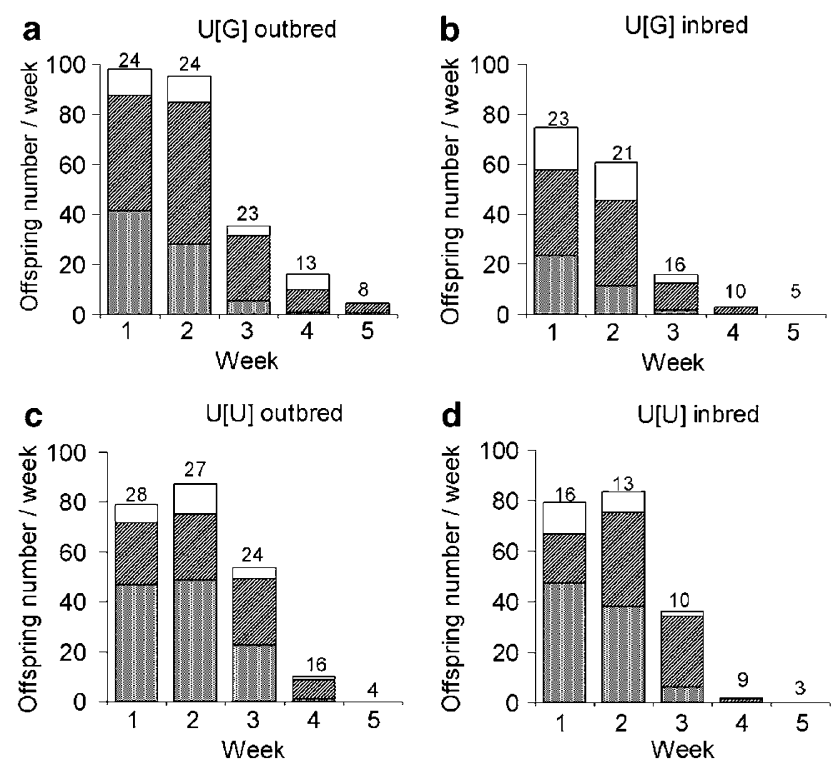

Figure 1 Lifetime offspring production of individual females from U[G] introgressed lines and U strain (U[U]), outbred or inbred (after five or six generations of full-sib mating). The mean number of offspring produced is split into females (dotted area), males (hatched area), and aborted eggs or dead larvae (clear area). Numbers above the diagrams are the numbers of females analyzed (one female per line). The decreasing number of females analyzed per week is either due to natural death or due to ending of oviposition before the end of the corresponding week.

weekly offspring production over the whole reproductive life (Figure 1). In U[G] females, inbreeding depression on fecundity was already expressed in the first week of oviposition and increased in intensity in the 2nd and 3rd weeks (Figure 1a, b). This pattern was apparent in a two-factor ANOVA with repeated measures (individual U[G] females), performed on weekly egg production: inbreeding $v s$ outbreeding status, week rank, and the interaction of both factors were all significant (whole model, $\quad \mathrm{F}_{55,113}=13.1, \quad P<0.0001 ;$ status, $\quad \mathrm{F}_{1,46}=8.4$,
$P=0.005$; week rank, $\mathrm{F}_{4,113}=124.6, P<0.0001$; interaction, $F_{4,113}=3.2, P=0.02$ ). The same analysis performed on control $U$ females did not reveal any significant effect of inbreeding vs outbreeding status, nor of an interaction with the timing of oviposition (week), despite a tendency for inbred females to produce fewer eggs, particularly between weeks 2 and 3 (Figure 1c, d). Thus, despite a marginally significant decrease in the lifetime fecundity of inbred $U$ females compared to outbred U females (Table 3), no effect of inbreeding on offspring production of $U$ females was detected on a weekly basis. This analysis, however, has low power because of the small number of $U$ inbred lines tested.

To follow the change in reproductive parameters of experimental U[G] lines in response to increased inbreeding levels, we measured fecundity and adult offspring production on the first 6 days of oviposition from the first to the 10th generation of full-sib mating. Fecundity decreased from the first to the fifth generation of full-sib mating in all introgressed lines except one (Figure 2a). Mean fecundity stabilized around 70-75 eggs/week thereafter (data not shown). By contrast, no decrease in fecundity with increasing inbreeding was observed in nonintrogressed $\mathrm{U}$ lines (Figure $2 \mathrm{~b}$ ).

\section{Discussion}

Introgression was initially performed to replace the cytoplasmic background of $T$. urticae $\mathrm{U}$ strain and compare the response of introgressed lines and nonintrogressed strain to inbreeding. A strong hybrid breakdown was observed in F1 females' fertility and egg viability, and was rescued by backcrossing to either parental strain (data not shown). Introgression increased hybrid fitness (viability and fertility) in four generations, suggesting that nuclear-nuclear and/or dominant cytonuclear negative epistatic interactions (Table 1, (2)) were causing hybrid breakdown (unpublished data). Since the present study on introgression and inbreeding effects was run long after hybrid rescue, there is no reason to believe that the mechanisms underlying the observed effects are the same as those involved in hybrid 
556

\section{a}

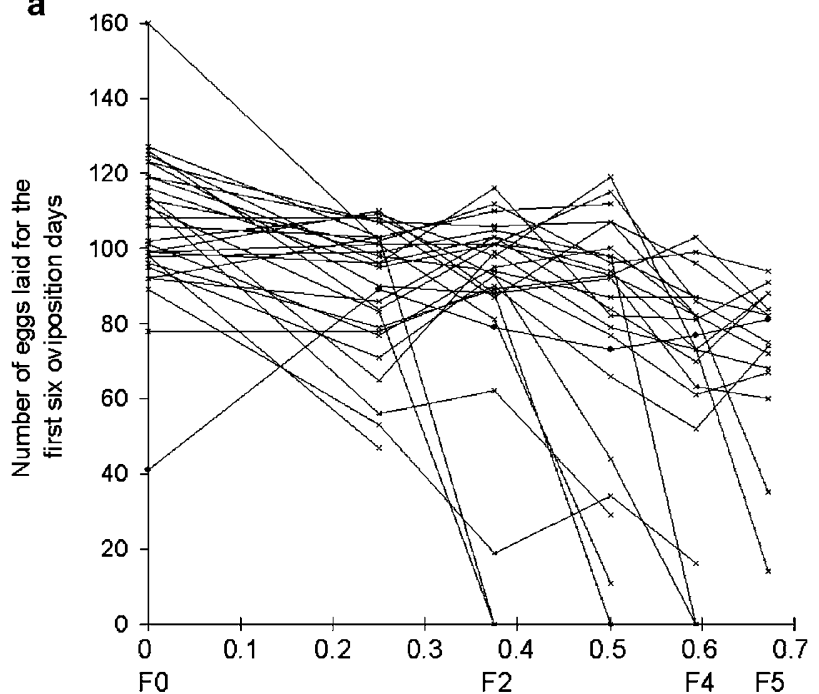

Inbreeding coefficient and number of inbreeding generations

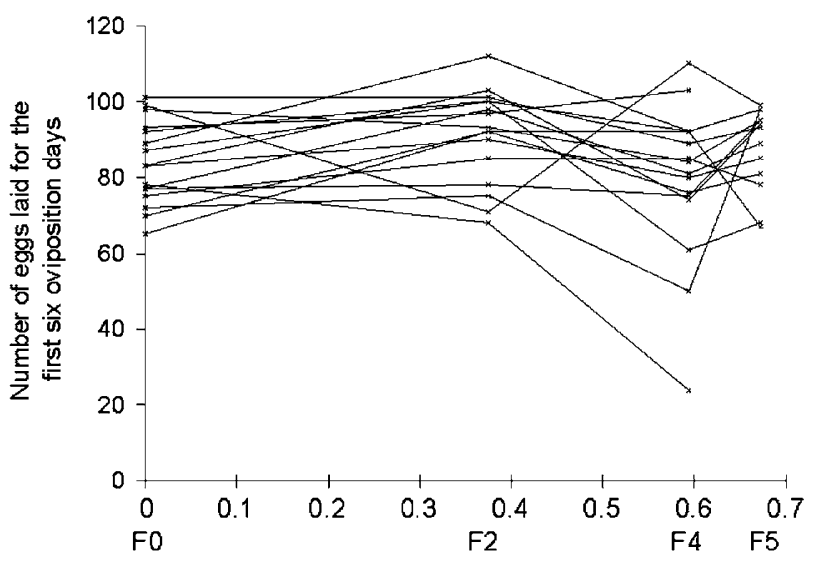

Inbreeding coefficient and number of inbreeding generations

Figure 2 Change in early female fecundity as in breeding proceeds in introgressed isofemale lines $\mathrm{U}[\mathrm{G}]$ and in control $\mathrm{U}$ lines. The number of eggs produced by one female per line over the first 6 days of oviposition is plotted against the inbreeding coefficient, for $29 \mathrm{U}[\mathrm{G}]$ lines (a) and 16 control $\mathrm{U}$ lines (b). Inbreeding via brother $\times$ sister mating was started after 27 generations of introgression in U[G] lines. Inbred U lines were each derived from one female from stock culture (F0) followed by brother $\times$ sister mating.

breakdown. In addition, the high number of generations of backcrosses to paternal strain $U$ prior to this study ( $>27$ ) should have restored some of the initial genetic variability of the paternal strain in introgressed lines, partly lost in the process of hybrid rescue and the associated strong bottleneck.

Significant effects of introgression on female reproductive parameters could be revealed due to contrasting patterns of egg production and sex ratio in the two parental strains $U$ and $G$. The lifetime fecundity of introgressed U[G] females was increased by $91 \%$ compared to the maternal strain $G$, to a level similar to the parental strain $U$. Increased offspring production in introgressed lines suggests that lifetime fecundity is determined by nuclear genes affecting oviposition rate and the length of oviposition period. The additional offspring produced were nearly exclusively of the male sex; hence the production of female offspring in U[G] females was maintained at the level of the maternal strain G. As a consequence, U[G] females produced a male-biased sex ratio (median and quartiles, 0.39 (0.26$0.52), n=51)$, in contrast with $\mathrm{U}$ and $\mathrm{G}$ females $(0.58(0.5-$ $0.72), n=55$ and $0.74(0.67-0.80), n=57$, respectively). One interpretation for increased male production is a sperm shortage, as unfertilized eggs can develop into males. However, where females had continuous access to multiple males, repeated mating would then have been expected to replenish sperm and restore the sex ratio, a pattern not observed here. Besides, sperm depletion is typically revealed in arrhenotokous insects and mites as a decrease in female production later in the oviposition period as sperm becomes depleted ( $\mathrm{Li}$ and Harmsen, 1993). In contrast, the pattern observed here shows a regularly high investment in male production from the first week of oviposition (Figure 1). This observation confirms previous data in tetranychid mites showing that the first mating is effective while the later ones are usually not (Helle and Overmeer, 1973). We can thus reject the hypothesis of sperm depletion as a cause of increased male production in the offspring of introgressed females. Alternatively, increased male offspring production in introgressed lines might result from the combined effects of cytoplasmically inherited elements controlling female offspring production and nuclear genes controlling fecundity. Maternally inherited cytoplasmic elements include mitochondria and intracellular microorganisms with exclusive vertical transmission. The two strains of T. urticae used here harbored Wolbachia endosymbionts, but infection had no effect on reproductive parameters and was not inducing cytoplasmic incompatibility (Perrot-Minnot et al, 2002). However, we could only definitely rule out the role of Wolbachia in the observed maternal effects on sex allocation in introgressed lines, by curing these lines of the bacteria. Other cytoplasmically inherited elements might also be involved in the control of female production. As female production in haplodiploid species is controlled by egg fertilization, cytoplasmic elements may affect either sperm access to the egg, or egg receptivity to the sperm. This interpretation is consistent with the prediction of a maternal control of sex ratio (Hamilton, 1967), if we assume that controlling daily female production is a way of adjusting the sex ratio. This holds if female production is adjusted to the oviposition rate, which may indeed occur in coadapted nuclear-cytoplasmic gene complexes. The maternal control of sex allocation has also received empirical support in haplodiploid insects and mites (Overmeer and Harrison, 1969). The involvement of cytoplasmic factors has been proposed (Overmeer and Harrison, 1969), a mechanism also hypothesized for sex determination in haplodiploid tetranychid mites based on several studies (Helle and Overmeer, 1973). A third explanation for a high male production in introgressed lines may be the partial sterility of U[G] female $\times U$ male crosses, possibly due to sperm-egg fertilization problems. These two last hypotheses cannot be easily disentangled, since they both involve cytoplasmic factors controlling either egg fertilization or sperm processing within the egg. It is interesting to note that an analysis of COI mtDNA sequences of T. urticae worldwide samples has shown that this species is highly structured, and includes a distinct Mediterranean lineage to which Greek 
populations belong (Navajas et al, 1998). Genetic polymorphism and strain clustering have also been found among Wolbachia infecting various T. urticae populations. In particular, partial wsp gene sequences of Wolbachia infecting $U$ and $G$ strains were distant by 20\% (PerrotMinnot et al, 2002). Thus, the genetic divergence of mitochondrial DNA between these two strains may be high enough to reveal, in introgressed lines, some maladaptation of cytoplasmic factors to the nuclear genome, or an underlying conflict between nuclear and cytoplasmic genes over sex allocation. Male-biased sex ratio is considered maladaptative, relative to a femalebiased evolutionary optimal sex ratio expected in haplodiploid species with partial inbreeding (Hamilton, 1967). Introgression thus has a negative fitness effect, expressed as a bias of sex ratio towards males, possibly due to the combined effects of nuclear genes on oviposition rate and cytoplasmic ones on female offspring production.

The nuclear and cytoplasmic effects on female reproduction reported here can be interpreted in two nonexclusive ways: (1) the effect of nuclear genes on oviposition rate and cytoplasmic genes on female offspring production act upon female reproductive phenotype independently, (2) both genomes (nuclear genes and either mtDNA or Wolbachia) interact in the expression of one or both of these reproductive traits, but the dominance of one set of genes on the other masked any nuclear-cytoplasmic interaction. To test for recessive nuclear-cytoplasmic interactions, we monitored the response of introgressed and control females to inbreeding. Compared to outbred females, female lifetime fecundity and egg viability were decreased by 40 and $80 \%$, respectively, in U[G] inbred females, whereas fecundity of $U$ females was decreased by $16 \%$ following inbreeding. Thus, cytoplasmic background has affected the intensity of inbreeding depression. In addition, the difference between outbred and inbred $\mathrm{U}[\mathrm{G}]$ females in lifetime fecundity and egg viability suggests that the effect of introgression was masked in outbred U[G] females. This gives evidence for an increased number of deleterious recessive interacting genes acting upon lifetime fecundity in introgressed females, resulting from maladaptation of nuclear and cytoplasmic genomes. The fact that both male and female egg production was reduced under inbreeding in introgressed U[G] females suggests that female control of egg fertilization occurred before prenatal egg viability was reduced. Reduced egg viability after oviposition can also be interpreted as an expression of early maternal effects in eggs from inbred females. Finally, the low but significant level of inbreeding depression on the fecundity of control $U$ females is in agreement with a much lower level of inbreeding depression in haplodiploids compared to diplodiploids, expected in insects and mites (reviewed by Henter, 2003). Inbreeding depression restricted to female-limited traits, for example, egg hatchability and fecundity, has been reported in Tetranychus species ((Helle, 1965; Helle and Overmeer, 1973) and in the subsocial haplodiploid mite Schizotetranychus miscanthi (Saito et al, 2000).

Although the present study aimed at investigating nuclear-cytoplasmic coadaptation, our data could also be relevant to the understanding of the genetic mechanisms underlying inbreeding depression. In fact, our results show that nuclear-cytoplasmic interactions may contribute to the genetic load. All recessive deleterious nuclear genes contribute to reduced fitness on inbreeding, whether they interact with the cytoplasm or not. Thus, the contribution of deleterious recessive nuclear alleles interacting with cytoplasmic ones to the inbreeding load will be confounded with other effects in most studies, unless the cytoplasmic background is manipulated.

\section{Acknowledgements}

We thank B Cheval for her help with introgression and crossing experiments. P Jarne and several anonymous reviewers made valuable comments on an earlier version of the manuscript.

\section{References}

Blier PU, Dufresne F, Burton RS (2001). Natural selection and the evolution of mtDNA-encoded peptides: evidence for intergenomic co-adaptation. Trends Genet 17: 400-406.

Breeuwer JAJ, Werren JH (1995). Hybrid breakdown between two haplodiploid species: the role of nuclear and cytoplasmic genes. Evolution 49: 705-717.

Crozier R (1985). Adaptive consequences of male haploidy. In: Helle W, Sabelis MW (eds) Spider Mites: Their Biology, Natural Enemies and Control, Elsevier: Amsterdam. pp 201-222.

Edmands S, Burton RS (1999). Cytochrome-c-oxydase activity in interpopulation hybrids of a marine copepod: a test for nuclear-nuclear or nuclear-cytoplasmic coadaptation. Evolution 53: 1972-1978.

Hamilton WD (1967). Extraordinary sex ratios. Science 156: 477-487.

Helle W (1965). Inbreeding depression in an arrhenotokous mite (Tetranychus urticae). Ent Exp Appl 8: 299-304.

Helle W, Overmeer WPJ (1973). Variability in tetranychid mites. Ann Rev Entomol 18: 97-120.

Henter H (2003). Inbreeding depression and haplodiploidy: experimental measures in a parasitoid and comparisons across diploid and haplodiploid insect taxa. Evolution 57: 1793-1803.

Li SY, Harmsen R (1993). Effects of maternal density and age on the daily fecundity and offspring sex ratio in Tetranychus urticae Koch. Can Entomol 125: 633-635.

Navajas M, Lagnel J, Gutierrez J, Boursot P (1998). Species-wide homogeneity of nuclear ribosomal ITS2 sequences in the spider mite Tetranychus urticae contrasts with extensive mitochondrial COI polymorphism. Heredity 80: 742-752.

Navajas M, Tsagkarakov A, Lagnel J, Perrot-Minnot M-J (2000). Genetic differentiation in Tetranychus urticae (Acari: Tetranychidae): polymorphism, host races or sibling species? Exp Appl Acarol 24: 365-376.

Overmeer WPJ, Harrison RA (1969). Notes on the control of the sex ratio in populations of the two-spotted spider mite, Tetranychus urticae Koch (Acarina: Tetranychidae). New Zeal J Zool 12: 920-928.

Perrot-Minnot M-J, Cheval B, Migeon A, Navajas M (2002). Contrasting effects of Wolbachia on cytoplasmic incompatibility and fecundity in the haplodiploid mite Tetranychus urticae. J Evol Biol 15: 808-817.

Roubertoux PL, Sluyter F, Carlier M, Marcet B, Maarouf-Veray F, Cherif C et al (2003). Mitochondrial DNA modifies cognition in interaction with the nuclear genome and age in mice. Nat Genet 35: 65-69.

Sackton TB, Haney RA, Rand DM (2003). Cytonuclear coadaptation in Drosophila: disruption of cytochrome $c$ oxidase activity in backcross genotypes. Evolution 57: 2315-2325. 
Saito Y, Sahara K, Mori K (2000). Inbreeding depression by recessive deleterious genes affecting female fecundity of a haplo-diploid mite. J Evol Biol 13: 668-678.

Siegel S, Castellan NJ (1988). Nonparametric Statistics for the Behavioural Sciences, 2nd edn. McGraw-Hill: Montreal.

Sokal RR, Rolf FJ (1995). Biometry, 3rd edn. Freeman WH Company: New York.
Werren JH (1993). The evolution of inbreeding in haplodiploid organisms. In: Thornhill NW (ed) The Natural History of Inbreeding and Outbreeding, Chicago Press: Chicago. pp 42-59.

Willett CS, Burton RS (2001). Viability of cytochrome C genotypes depends on cytoplasmic backgrounds in Tigriopus californicus. Evolution 55: 1592-1599. 